Received: 2020/09/06, Revised: 2020/12/04, Accepted: 2020/12/11, Published: 2020/12/31 (๑2020 Satoshi Hattori et al.; Licence Physical Activity and Nutrition. This is an open access article distributed under the terms of the creative commons attribution license (https:/l creativecommons.org/licenses/by-nc/2.0/), which permits unrestricted use, distribution, and reproduction in any medium, provided the orginal work is properly cited.

${ }^{*}$ Corresponding author : Naomi Omi

Faculty of Health and Sport Sciences, University of Tsukuba, 1-1-1 Tennodai, Tsukuba, Ibaraki 305-8572, Japan.

Tel: +81-29-853-6319

Fax: +81-29-853-6507

E-mail: omi.naomi.gn@u.tsukuba.ac.jp

@22020 The Korean Society for Exercise Nutrition
[Purpose] Dehydroepiandrosterone (DHEA) administration reportedly recovers osteoporosis, a bone disorder associated with bone deficiency in postmenopausal women. However, the physiological mechanism of DHEA in osteoporosis remains elusive, especially in terms of intestinal calcium absorption. Therefore, we investigated the effect of DHEA administration on calcium absorption in ovariectomized (OVX) female rats using an estrogen receptor antagonist.

[Methods] Female Sprague-Dawley rats $(\mathrm{n}=23,6$ weeks old) were randomized into three groups: OVX control group (OC, $n=7)$, OVX with DHEA treatment group (OD, $n=8)$, and OVX with DHEA inhibitor group $(\mathrm{ODI}, \mathrm{n}=8)$ for 8 weeks.

[Results] Intestinal calcium accumulation, as well as the rate of absorption, demonstrated no significant differences during the experimental period among investigated groups. The bone mineral density (BMD) of the tibia at the proximal metaphysis was higher in the OD group than that in the OC group $(p<0.05)$; however, BMD of the ODI group showed no significant difference from investigated groups. Furthermore, the BMD of the tibia at the diaphysis did not significantly differ among these groups.

[Conclusion] We revealed that DHEA administration does not involve intestinal $\mathrm{Ca}$ absorption, although this treatment improves $\mathrm{BMD}$ levels in OVX rats. These observations indicate that the effect of DHEA on the bone in postmenopausal women is solely due to its influence on bone metabolism and not intestinal calcium absorption.

[Key words] DHEA, Calcium absorption, Osteoporosis, BMD, OVX, Fulvestrant

\section{The effect of dehydroepiandrosterone administration on intestinal calcium absorption in ovariectomized female rats}

Satoshi Hattori ${ }^{1}$ / Suhan Park ${ }^{2}$ / Jong-hoon Park ${ }^{3}$ / Naomi Omi1 ${ }^{\text {* }}$

1. Faculty of Health and Sport Sciences, University of Tsukuba, Ibaraki, Japan

2. Comprehensive Human Sciences, University of Tsukuba, Ibaraki, Japan

3. Department of Physical Education, Korea University, Seoul, Republic of Korea

\section{INTRODUCTION}

Bone is a hard tissue mainly composed of hydroxyapatite in vertebrate animals. This organ protects soft tissues against outside mechanical stress, exercising the peripheral tissues owing to the principle of leverage. Reportedly, in most individuals, the onset of bone disorders is characterized by low bone mineral density (BMD) and deterioration of trabecular bone microarchitecture, namely osteoporosis ${ }^{1}$. This disease increases a person's risk of fracture owing to bone fragility, along with increased medical costs. Osteoporosis is often caused by sex hormone deficiencies, with the crisis rate of osteoporosis dramatically elevated, especially in postmenopausal women.

Sex hormones (e.g., estrogen and androgen) play important roles in bone remodeling, primarily by inhibiting bone breakdown. It is well known that estrogen levels in postmenopausal women are generally decreasing, and reduced estrogen causes rapid bone loss. Therefore, estrogen replacement therapy has been widely accepted for the prevention of osteoporosis in high-risk postmenopausal women ${ }^{2}$. However, according to the Women's Health Initiative study, this therapy is reportedly associated with adverse events such as cardiovascular disease, stroke, and invasive breast cancer ${ }^{3}$.

Dehydroepiandrosterone (DHEA) is mainly a secretory product of the adrenal gland and ovary, which is a crucial precursor of sex hormones and exerts indirect endocrine and intracrine actions on various peripheral tissues ${ }^{4,5}$. Exogenous DHEA treatment has estrogenic effects in postmenopausal women, thereby improving menopausal symptoms (e.g., abnormal energy metabolism, bone loss, and decline of cognitive performance $)^{6}$. Furthermore, DHEA administration abolishes the reduced BMD in ovariectomized (OVX) animals ${ }^{7}$. However, the mechanisms through which DHEA increases BMD are not well understood. In a previous study, we investigated the effect of DHEA administration on intestinal calcium $(\mathrm{Ca})$ absorption in OVX rats; however, we failed to observe a relationship between DHEA and the intestinal system ${ }^{8}$. These results could be explained as follows 1) DHEA administration might in- 
crease intestinal $\mathrm{Ca}$ absorption during an earlier phase of the 8-week experimental period, 2) the lack of a DHEA effect could be attributed to the $1.05 \% \mathrm{Ca}$ diet employed, which is a sufficient $\mathrm{Ca}$ amount for maintaining bone health in OVX rats. Therefore, we designed a time-course study under $\mathrm{Ca}$ restriction to investigate the influence of DHEA treatment on intestinal $\mathrm{Ca}$ absorption.

\section{METHODS}

\section{Experimental animals and conditions}

Female Sprague-Dawley rats $(n=23,6$ weeks old) were purchased from CLEA Japan (CE-2, CLEA Japan, Inc., Japan) and were acclimatized for 6 days. Then, the rats were surgically OVX and randomized into three groups: OVX control group (OC, $n=7)$, OVX with DHEA treatment group (OD, $\mathrm{n}=8$ ), and OVX with DHEA inhibitor group (ODI, $\mathrm{n}=8)$. The rats were fed a low calcium diet $(0.3 \%$ calcium, $0.3 \%$ phosphate) throughout the experimental period. DHEA ( $20 \mathrm{mg} / \mathrm{kg}$ body weight) and an estrogen receptor antagonist (DHEA inhibitor: $1 \mathrm{mg} / \mathrm{kg}$ body weight, fulvestrant, Sigma, USA), dissolved in sesame oil $(0.5 \mathrm{~mL})$, were intraperitoneally administrated to the OD and ODI groups once every 3 days for 8 weeks, as described in our previous study ${ }^{9}$. The OC group was treated with sesame oil only. All rats were housed in individual single cages, with access to food and distilled water ad libitum. Food consumption and body weight gain were measured every second day. The room was maintained at $23 \pm 1{ }^{\circ} \mathrm{C}$, with $50 \pm 5 \%$ humidity, and under a 12:12 h light-dark cycle (light 8:00 to 20:00). Animal care and experimental procedures were approved by the Experimental Animal Committee of the University of Tsukuba. All rats were fasted on the day before tissue isolation. Following euthanasia, the tibias were collected and stored in $70 \%$ ethanol after the soft tissue was carefully removed. Animal care and experimental procedures were approved by the Experimental Animal Committee of the University of Tsukuba.

\section{BMD measurement}

The BMD of isolated tibias was evaluated using dualenergy X-ray absorptiometry (DXA; Aloka, DCS-600R Tokyo, Japan) as described in a previous study ${ }^{10}$. The tibia was categorized into five divisions. The first division was considered the proximal metaphysis site and was mainly composed of the cortical bone. The second to third divisions were regarded as the diaphysis site.

\section{Ca balance}

We evaluated the $\mathrm{Ca}$ balance ( $\mathrm{Ca}$ absorption and $\mathrm{Ca}$ accumulation) in experimental rats as described in our previous study ${ }^{8}$. Briefly, animals were placed in metabolic cages to collect 24-h urine and feces samples at each time point (Metabolic cage phase (MC) 1: 2 days before initiating experimental conditions; MC2: 2 days immediately after initiating the experiment; MC3-6: every 10 days after initiating the experiment). Urine samples were collected in bottles containing $1 \mathrm{~mL}$ of $2 \mathrm{~N}$ hydrochloric acid to prevent Ca precipitation, followed by centrifugation $(2,500 \mathrm{rpm}, 15$ $\mathrm{min})$ to extract the supernatant. The urine Ca levels were evaluated by inductively coupled plasma atomic emission spectroscopy (ICAP-AES-575v, Nippon Jarrell-Ash). Fecal samples were incinerated in a muffle furnace at $550-600^{\circ} \mathrm{C}$ for approximately $15 \mathrm{~h}$, and fecal Ca was measured using a method similar to that for urinary estimation. Intestinal $\mathrm{Ca}$ accumulation was calculated using the following formula: intestinal $\mathrm{Ca}$ accumulation $=\mathrm{Ca}$ intake - fecal $\mathrm{Ca}$ discharge - urinary $\mathrm{Ca}$ discharge.

\section{Statistics}

All data are expressed as the mean \pm standard error (SE) and were analyzed using SPSS (version 18.0 J; SPSS Inc., Chicago, IL). One-way analysis of variance (ANOVA) was used to test statistically significant differences among groups. If a significant difference was detected, these groups were further evaluated using Fisher's F-test for multiple comparisons. The significance level for major effects was established at $\mathrm{p}<0.05$.

\section{RESULTS}

Final body weight, body weight gain, food intake, and food efficiency are shown in Table.1. The final body weight was significantly lower in the OD group than that in the OC group $(\mathrm{p}<0.01)$. Body weight gain was significantly lower in the OD and ODI groups than that in the OC group ( $<<0.001)$. Additionally, food intake and food efficiency did not significantly differ among these groups (Table 1).

Table 1. Body weight and Food intake.

\begin{tabular}{lcccc} 
& $\begin{array}{c}\text { Final } \\
\text { body weight } \\
\text { (g) }\end{array}$ & $\begin{array}{c}\text { Body } \\
\text { weight gain } \\
\text { (g/day) }\end{array}$ & $\begin{array}{c}\text { Food intake } \\
\text { (g/day) }\end{array}$ & $\begin{array}{c}\text { Food } \\
\text { efficiency } \\
\text { (\%) }\end{array}$ \\
OC & $401.4 \pm 7.2$ & $3.6 \pm 0.11$ & $18.4 \pm 0.42$ & $0.19 \pm 0.01$ \\
OD & $346.0 \pm 9.2^{* *}$ & $2.6 \pm 0.12^{* *}$ & $17.2 \pm 0.39$ & $0.15 \pm 0.00$ \\
ODI & $365.1 \pm 15.8$ & $2.6 \pm 0.25^{* *}$ & $17.7 \pm 0.79$ & $0.16 \pm 0.01$ \\
\hline
\end{tabular}

Data show mean \pm SE. OC: Ovariedtomy Control group, OD: Ovariectomy with DHEA treatment group, ODI: Ovariedtomy with DHEA Inhibitor group. ${ }^{* *} p<0.01,{ }^{* * *} p<0.001$ vs OC.

(DHEA, dehydroepiandrosterone)

At each time point assessed (MC1-6, Fig. 1A), intestinal $\mathrm{Ca}$ accumulation did not significantly differ, and the $\mathrm{Ca}$ accumulation rate was not significantly different between the two groups (Fig. 1B). Furthermore, urinary Ca discharge did not differ (Fig. 1C), similar to the results for fecal Ca discharge (data not shown).

The BMD of the tibia at the proximal metaphysis was higher in the OD group than that in the OC group $(\mathrm{p}<0.05)$, but that of the ODI group did not significantly different from the two groups (Fig. 2A). In contrast, the BMD of the tibia at the diaphysis did not significantly differ between investigated groups (Fig. 2B). 
A)

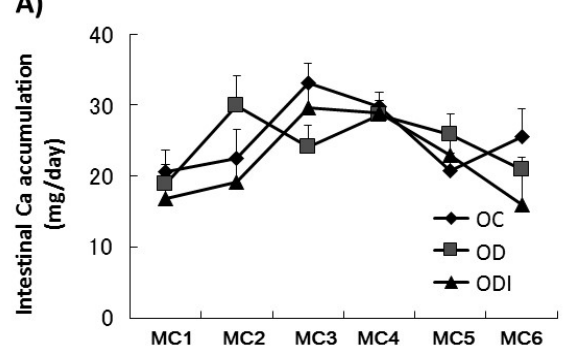

B)
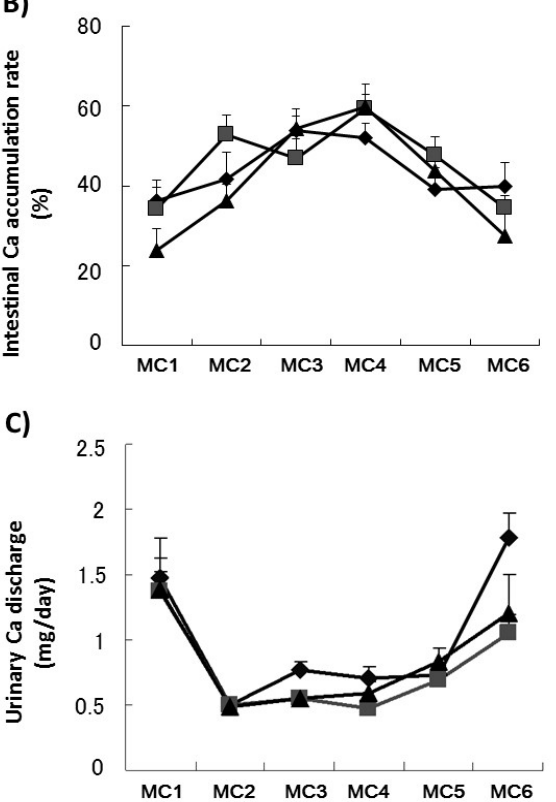

Figure 1. Intestinal $\mathrm{Ca}$ absorption (A), rate of $\mathrm{Ca}$ absorption (B), and urinary Ca discharge (C). Metabolic cage phase (MC) 1 was performed 2 days before starting experimental conditions, MC2 was performed 2 days immediately after experiment initiation, and MC3-6 was performed every 10 days after experiment initiation. Samples were collected over a 24-h period. OVX Control group (OC, $n=7)$, OVX with DHEA treatment group (OD, $n=8)$, and OVX with DHEA inhibitor group (ODI, $n=8)$. Data show mean \pm standard error $(S E)$. Ca, calcium; OVX, ovariectomized; DHEA, dehydroepiandrosterone.

\section{DISCUSSION}

Osteoporosis is a metabolic bone disease characterized by bone loss and deterioration of the bone structure, resulting in enhanced pain, exercise disability, increased total health care cost, and mortality, especially in postmenopausal women. Accordingly, DHEA administration may be an effective approach in this disease if DHEA upregulates intestinal $\mathrm{Ca}$ accumulation. In the present study, our findings indicate that DHEA administration did not affect intestinal $\mathrm{Ca}$ accumulation or the rate of $\mathrm{Ca}$ accumulation; however, it rescued tibial BMD levels in OVX female rats during calcium restriction. These findings revealed that DHEA treatment did not influence intestinal $\mathrm{Ca}$ absorption.

Several studies have reported that E2 treatment recovered intestinal $\mathrm{Ca}$ and bone loss absorption in both OVX animals
A)

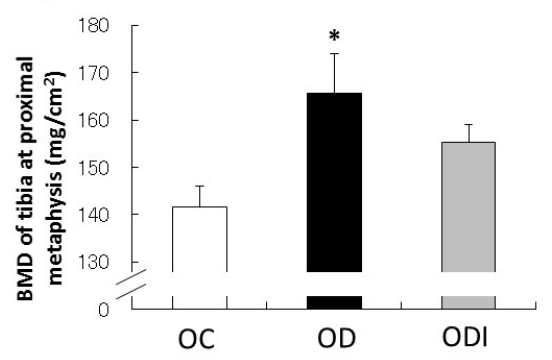

B)

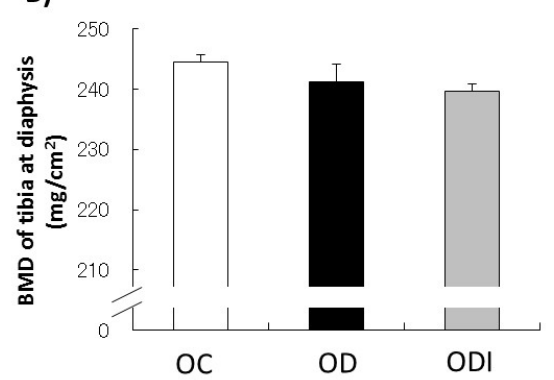

Figure 2. $B M D$ of the tibia at proximal metaphysis $(A)$, and diaphysis (B). BMD levels were measured by DXA. OVX Control group (OC, $n=7$ ), OVX with DHEA treatment group (OD, $n=8)$, and OVX with DHEA inhibitor group (ODI, $n=8)$. * $p<0.05$ vs OC. Data show mean \pm standard error (SE). BMD, bone mineral density; OVX, ovariectomized; DHEA, dehydroepiandrosterone; DXA, dual-energy X-ray absorptiometry.

and postmenopausal women ${ }^{11,12}$. However, in the present study and a previous report ${ }^{9}$, DHEA administration did not recover intestinal $\mathrm{Ca}$ absorption and urinary $\mathrm{Ca}$ discharge, while increasing BMD. The reason underlying the recovery effects observed solely on BMD could be because these tissues are mainly affected by DHEA via different pathways. DHEA is converted to testosterone and further metabolized to dihydrotestosterone in osteoblasts, and can inhibit bone loss in OVX rats ${ }^{13}$. We have previously revealed that the same amount of DHEA treatment prevents bone deficiency of the tibia via androgen metabolism in OVX rats ${ }^{8}$. Conversely, DHEA is also metabolized to E2, which affects the intestine. However, the conversion volume of this hormone might be below the threshold level necessary to elevate intestinal $\mathrm{Ca}$ absorption in the present study. Therefore, the effect of DHEA on bone volume may be mediated mainly through the direct pathway in the bone.

In the OD group, the BMD level of the proximal metaphysis of the tibia was rescued by DHEA administration, whereas this effect was not observed in the diaphysis of the tibia. DHEA administration generally leads to increased BMD in OVX rats ${ }^{14}$. Reportedly, the trabecular bone (mainly composing the proximal metaphysis) is more sensitive to mechanical loading than cortical bone (mainly composing the diaphysis) ${ }^{15,16}$. Furthermore, our previous study similarly revealed this phenomenon ${ }^{8}$. Therefore, these results may be attributed to the body weight difference between the two groups as the site of the proximal metaphysis is more responsible for mechanical stress than that of the diaphysis.

DHEA administration decreases body weight by enhanc- 
ing the utilization of ingested energy in the brown adipose tissue; therefore, DHEA was considered to have an anti-obesity effect in a rodent model of obesity ${ }^{17}$. In the present study, the final body weight was dramatically decreased in the OD group when compared with the OC group. Thus, this result suggests that DHEA administration elevates the energy metabolic system in the OD group.

This study had two limitations. First, the effects of other organ factors were not determined. Organs demonstrate inter-organ communication through some endocrine factors; thereby, bone metabolism is affected by other organs such as the skeletal muscle and adipose tissues ${ }^{18}$. In the present study, we did not sample these tissues or evaluate the condition of these tissues. Thus, the impact of other organs involved in bone metabolism remains unknown. Second, bone turnover was not evaluated in the present study. We did not measure extracellular markers of bone metabolism in experimental animals (e.g., osteocalcin and osteopontin). Therefore, bone formation and resorption were not determined, although the bone phenotypes were evaluated by BMD levels.

In conclusion, we demonstrated that DHEA administration does not involve intestinal $\mathrm{Ca}$ absorption. These findings suggest that the effect of DHEA on bone mass maintenance in postmenopausal women is solely attributed to its influence on bone metabolism, and other mechanisms are needed to improve intestinal $\mathrm{Ca}$ absorption. Therefore, an intervention that combines DHEA administration and other treatments to enhance intestinal $\mathrm{Ca}$ absorption is needed to maintain bone health in these subjects.

\section{ACKNOWLEDGEMENTS}

We would like to thank the members of the exercise and nutrition laboratory at the University of Tsukuba for useful discussions and support.

\section{REFERENCES}

1. Tu KN, Lie JD, Wan CKV, Cameron M, Austel AG, Nguyen JK, Van K, Hyun D. Osteoporosis: a review of treatment options. $P t$. 2018;43:92-104.

2. Klein KO, Rosenfield RL, Santen RJ, Gawlik AM, Backeljauw PF, Gravholt CH, Sas TCJ, Mauras N. Estrogen replacement in turner syndrome: literature review and practical considerations. J Clin Endocrinol Metab. 2018;103:1790-803.

3. Rossouw JE, Anderson GL, Prentice RL, LaCroix AZ, Kooperberg C, Stefanick ML, Jackson RD, Beresford SA, Howard BV, Johnson $\mathrm{KC}$, Kotchen JM, Ockene J. Risks and benefits of estrogen plus progestin in healthy postmenopausal women: principal results from the women's health initiative randomized controlled trial. JAMA. 2002;288:321-33.

4. Allolio B, Arlt W. DHEA treatment: myth or reality? Trends Endocrinol Metab. 2002;13:288-94.

5. Wang L, Wang YD, Wang WJ, Li DJ. Differential regulation of dehydroepiandrosterone and estrogen on bone and uterus in ovari- ectomized mice. Osteoporos Int. 2009;20:79-92.

6. Davis SR, Panjari M, Stanczyk FZ. Clinical review: DHEA replacement for postmenopausal women. J Clin Endocrinol Metab. 2011;96:1642-53.

7. Turner RT, Lifrak ET, Beckner M, Wakley GK, Hannon KS, Parker LN. Dehydroepiandrosterone reduces cancellous bone osteopenia in ovariectomized rats. Am J Physiol. 1990;258:e673-7.

8. Park J, Omi N. DHEA administration has limited effect onintestinal Ca absorption in ovariectomized rats. J Exerc Nutrition Biochem. 2014;18:333-7.

9. Park JH, Aizawa K, lemitsu M, Sato K, Akimoto T, Agata U, Maeda S, Ezawa I, Omi N. DHEA administration activates local bioactive androgen metabolism in cancellous site of tibia of ovariectomized rats. Calcif Tissue Int. 2011;89:105-10.

10. Omi N, Goseki M, Oida S, Sasaki S, Ezawa I. The nutritional evaluation of globin on maintenance of bone metabolism in ovariectomized osteoporotic rats. J Nutr Sci Vitaminol (Tokyo). 1994:40:443-57.

11. Ten Bolscher M, Netelenbos JC, Barto R, Van Buuren LM, Van der vijgh WJ. Estrogen regulation of intestinal calcium absorption in the intact and ovariectomized adult rat. J Bone Miner Res. 1999;14:1197-202.

12. Gallagher JC, Riggs BL, DeLuca HF. Effect of estrogen on calcium absorption and serum vitamin $\mathrm{D}$ metabolites in postmenopausal osteoporosis. J Clin Endocrinol Metab. 1980;51:1359-64.

13. Coxam V, Bowman BM, Mecham M, Roth CM, Miller MA, Miller SC. Effects of dihydrotestosterone alone and combined with estrogen on bone mineral density, bone growth, and formation rates in ovariectomized rats. Bone. 1996;19:107-14.

14. Jankowski CM, Gozansky WS, Schwartz RS, Dahl DJ, Kittelson JM, Scott SM, Van Pelt RE, Kohrt WM. Effects of dehydroepiandrosterone replacement therapy on bone mineral density in older adults: a randomized, controlled trial. J Clin Endocrinol Metab. 2006:91:2986-93

15. Ott SM. Cortical or trabecular bone: what's the difference? Am J Nephrol. 2018:47:373-5

16. Cohen A, Dempster DW, Müller R, Guo XE, Nickolas TL, Liu XS, Zhang XH, Wirth AJ, van Lenthe GH, Kohler T, McMahon DJ, Zhou H, Rubin MR, Bilezikian JP, Lappe JM, Recker RR, Shane E. Assessment of trabecular and cortical architecture and mechanical competence of bone by high-resolution peripheral computed tomography: comparison with transiliac bone biopsy. Osteoporos Int. 2010;21:263-73.

17. Ryu JW, Kim MS, Kim CH, Song KH, Park JY, Lee JD, Kim JB, Lee KU. DHEA administration increases brown fat uncoupling protein 1 levels in obese OLETF rats. Biochem Biophys Res Commun. 2003;303:726-31.

18. Karsenty G, Olson EN. Bone and muscle endocrine functions: unexpected paradigms of inter-organ communication. Cell. 2016;164:1248-56 